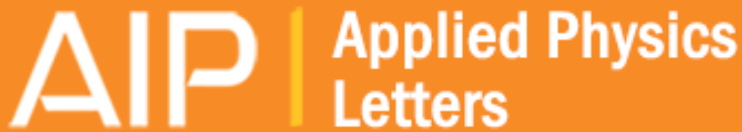

\section{Tensile strain in Ge membranes induced by SiGe nanostressors}

Michael R. Barget, Mario Lodari, Mauro Borriello, Valeria Mondiali, Daniel Chrastina, Monica Bollani, and

Emiliano Bonera

Citation: Applied Physics Letters 109, 133109 (2016); doi: 10.1063/1.4963657

View online: http://dx.doi.org/10.1063/1.4963657

View Table of Contents: http://scitation.aip.org/content/aip/journal/apl/109/13?ver=pdfcov

Published by the AIP Publishing

\section{Articles you may be interested in}

Local uniaxial tensile strain in germanium of up to $4 \%$ induced by SiGe epitaxial nanostructures

Appl. Phys. Lett. 107, 083101 (2015); 10.1063/1.4928981

Strain-induced wrinkling on SiGe free standing film

Appl. Phys. Lett. 89, 043119 (2006); 10.1063/1.2236299

Hydrogen gettering and strain-induced platelet nucleation in tensilely strained Si 0.4 Ge 0.6 /Ge for layer exfoliation applications

J. Appl. Phys. 97, 104511 (2005); 10.1063/1.1900928

Optical properties of strain-balanced SiGe planar microcavities with Ge dots on Si substrates

Appl. Phys. Lett. 81, 817 (2002); 10.1063/1.1496142

Controlling threading dislocation densities in Ge on Si using graded SiGe layers and chemical-mechanical polishing

Appl. Phys. Lett. 72, 1718 (1998); 10.1063/1.121162

\section{Pure Metals • Ceramics} Alloys • Polymers in dozens of forms
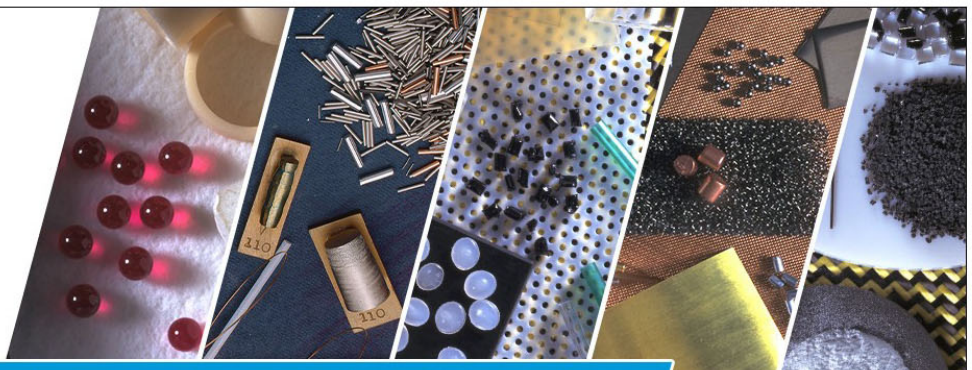


\title{
Tensile strain in Ge membranes induced by SiGe nanostressors
}

\author{
Michael R. Barget, ${ }^{1, \text { a) }}$ Mario Lodari, ${ }^{2,3}$ Mauro Borriello, ${ }^{2}$ Valeria Mondiali, ${ }^{2}$ \\ Daniel Chrastina, ${ }^{2}$ Monica Bollani, ${ }^{3}$ and Emiliano Bonera ${ }^{1, a)}$ \\ ${ }^{1}$ Dipartimento di Scienza dei Materiali and L-NESS, Università di Milano-Bicocca, via Cozzi 55, \\ I-20125 Milano, Italy \\ ${ }^{2}$ L-NESS and Dipartimento di Fisica, Politecnico di Milano, Polo di Como, via Anzani 42, 22100 Como, Italy \\ ${ }^{3}$ IFN-CNR, L-NESS, via Anzani 42, 22100 Como, Italy
}

(Received 9 June 2016; accepted 14 September 2016; published online 28 September 2016)

\begin{abstract}
The monolithic integration of photonic functionality into silicon microtechnology is widely advanced. Yet, there is no final solution for the realization of a light source compatible with the prevailing complementary metal-oxide-semiconductor technology. A lot of research effort focuses on germanium $(\mathrm{Ge})$ on silicon $(\mathrm{Si})$ heterostructures and tensile strain application to $\mathrm{Ge}$ is accepted as one feasible route to make Ge an efficient light emitter. Prior work has documented the special suitability of $\mathrm{Ge}$ membranes to reach the high tensile strain. We present a top-down approach for the creation of SiGe stressors on Ge micro-bridges and compare the obtained strain to the case of an attached bulk-like Ge layer. We could show that the Ge influenced by a SiGe stressor is under tensile strain; absolute strain values are of the order of $0.7 \%$ for both micro-bridge and bulk. The relative strain induced by the nanostructures in the micro-bridge is $1.3 \%$ due to the high sharing of elastic energy between nanostructures and bridges. Published by AIP Publishing.

[http://dx.doi.org/10.1063/1.4963657]
\end{abstract}

The monolithic integration of photonic functionality into silicon microtechnology is widely advanced with the demonstration of optical modulators ${ }^{1}$ and photodetectors. ${ }^{2,3}$ Silicon itself is an inefficient light emitter due to its indirect fundamental electronic band gap and there is no solution as yet for the realization of a monolithically integrable light source. A lot of research effort focuses on germanium $(\mathrm{Ge})$ on silicon ( $\mathrm{Si}$ ) heterostructures, since $\mathrm{Ge}$ is compatible with the standard complementary metal-oxide-semiconductor (CMOS) technology. ${ }^{4}$ Moreover, its electronic band structure features a local minimum at the $\Gamma$ point which is only about $140 \mathrm{meV}$ higher in energy than the overall minimum at L. ${ }^{5}$ However, only about $\sim 10^{-4}$ of excited electrons will be in the direct gap valley at $\Gamma$ due to the much larger density of states in the four-fold degenerate L valleys. ${ }^{6}$ In addition to tensile strain to reduce the $\Gamma-\mathrm{L}$ energy difference, high levels of n-type doping can be applied to pre-fill the $\mathrm{L}$ valley with extrinsic carriers and thus raise the Fermi level in order to populate the $\Gamma$ valley. ${ }^{7,8}$ Optical gain ${ }^{9}$ and lasing from Ge under optical ${ }^{10}$ and electrical ${ }^{11,12}$ pumping have been demonstrated. However, only slightly strained ${ }^{13}$ or even unstrained materials have been used. The presented lasing devices suffered from high threshold current densities and low efficiencies. This can be traced back to high absorption losses (of excited carriers through Auger recombination as well as emitted photons by free-carrier absorption), due to the high doping concentration and the strong pumping required. ${ }^{8,14}$ In this regard, improvements can be made by decreasing the energy barrier between $\mathrm{L}$ and $\Gamma$. In fact, the doping concentration required for lasing can be reduced dramatically under increased tensile strain. ${ }^{15-17}$ Hence, it is of vital interest to introduce tensile strain in the active Ge

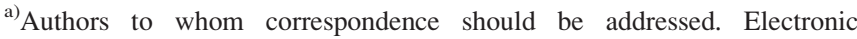
addresses: m.barget@campus.unimib.it and emiliano.bonera@unimib.it
}

region. Various methods have been applied in order to induce tensile strain in Ge. They comprise the use of plane stressor layers on grounded ${ }^{18-20}$ as well as on suspended Ge structure $^{21-24}$ and the exploitation of a strain accumulation effect by shaping free standing pre-strained Ge layers. ${ }^{25-28}$ From these results, as well as from simulations, ${ }^{29}$ it is clear that higher strain values can be obtained when using suspended Ge detached from a substrate.

In this work, we transfer our successful method ${ }^{20}$ from bulk material to suspended micro-bridges, using a patterned $\mathrm{SiGe}$ stressor layer to induce tensile strain in a suspended $\mathrm{Ge}$ layer. SiGe is grown pseudomorphically on $\mathrm{Ge}$, such that it is under tensile strain due to its smaller lattice constant with respect to Ge. Patterning creates free side walls which allow the SiGe layer to elastically relax, thereby compressing the Ge beneath. ${ }^{30}$ However, tensile strain is induced in the Ge inside a trench of the pattern, where SiGe was removed. Applying this technique to bulk Ge has led to up to $4 \%$ tensile strain for trenches of $20 \mathrm{~nm} .{ }^{20}$ The same strain level can be achieved with wider SiGe trenches on a Ge bridge. This enlarges the volume of strained Ge and thus increases the active area for both emission and detection applications. In the following, we present our first results of SiGe stressors on Ge micro-bridges and compare the obtained strain to the case of attached bulk-like Ge.

The $\mathrm{SiGe} / \mathrm{Ge}$ stack was grown by low-energy plasmaenhanced chemical vapor deposition (LEPECVD) on a $\mathrm{Si}$ (001) substrate. ${ }^{31}$ Bridge and stressors are then fabricated by e-beam lithography and reactive ion etching (RIE). Potassium hydroxide $(\mathrm{KOH})$ is then used to suspend the $\mathrm{SiGe} / \mathrm{Ge}$ bridge. The actual samples were processed from a stack of nominally $50 \mathrm{~nm} \mathrm{SiGe} \mathrm{with} 60 \%$ Ge content on $100 \mathrm{~nm} \mathrm{Ge}$ on $\mathrm{Si}(001)$ so that the SiGe stressor layer has a comparable thickness to the subsequently formed Ge bridge, see Fig. 1(a). Material composition and strain were determined by $\mathrm{x}$-ray diffraction 
(a)
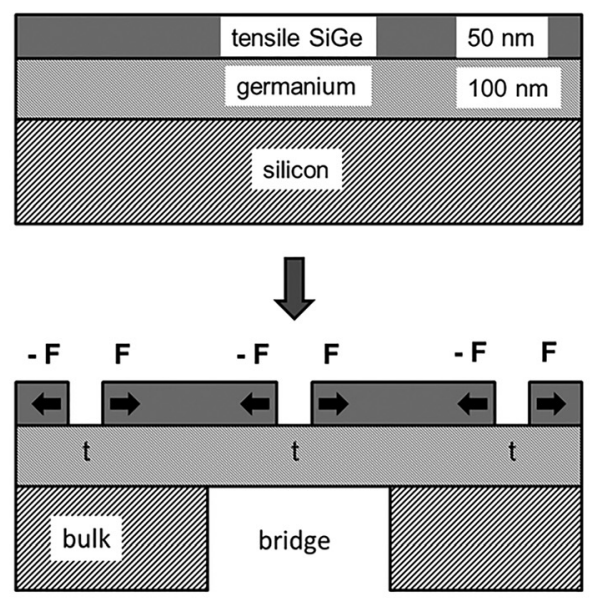

(b)

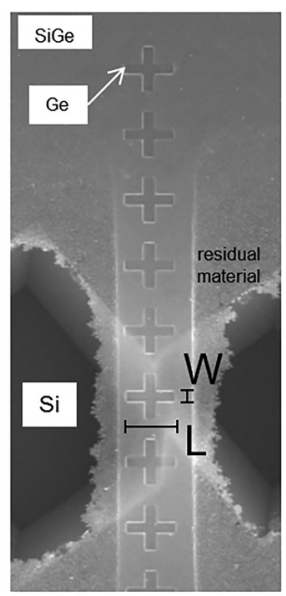

(c)

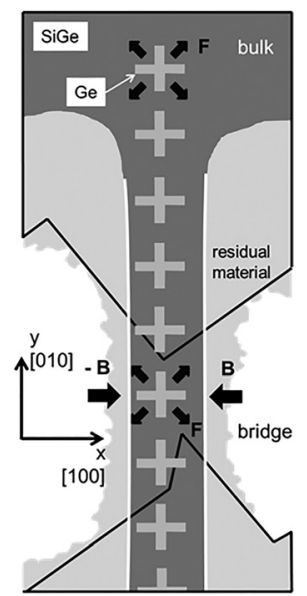

FIG. 1. (a) Sketches in cross section to illustrate the fabrication and working principle of the investigated system. The cross section is through the arms of the realized cross shaped patterns inside the SiGe layer. The perimeter forces at the side walls of the trenches (indicated by arrows F) pull the Ge inside a trench resulting in tensile-strained Ge (indicated with the letter "t"). The sample features stressor structures on the grounded ("bulk") and the free standing ("bridge") Ge material. (b) SEM image of the fabricated sample. The free standing part can be recognized by the lighter color; (c) sketch in top view. The SiGe/Ge bridge is not constrained in the [100] direction, resulting in a compressive strain bias as indicated with arrows B. Arrows F illustrate the action of the cross stressors and are equivalent to the arrows in (a).

(XRD). The Ge layer is slightly contaminated by $\mathrm{Si}$ (Ge content $98.6 \%$ ) and exhibits an in-plane strain of $\varepsilon_{\|}=0.05 \%$ due to partial relaxation of thermally induced strain. The SiGe layer has a Ge content of $59.7 \%$ and the in-plane strain is determined to be $\varepsilon_{\|}=1.64 \%$. This strain value indicates coherent growth on $\mathrm{Ge}$ and corresponds to a stress of $\sigma_{\|}=2.58 \mathrm{GPa}$. The creation of suspended $\mathrm{SiGe} / \mathrm{Ge}$ bridges and the stressor pattern in the SiGe layer are described in detail in Refs. 24 and 30. A key step for proper action of the stressor is that the etching of the trenches should stop exactly at the $\mathrm{SiGe} / \mathrm{Ge}$ interface, in order to maximize the local stress applied to the Ge. Fig. 1(b) shows the scanning electron micrograph (SEM) of a fully processed Ge bridge with cross stressors engraved into the SiGe top layer. The stressors on the suspended bridge can be recognized close to the center of the image (lighter color of suspended material), while at its ends the stressors on the bulk-like Ge can be seen. Fig. 1(c) depicts a sketch in top view and provides the crystallographic orientation of the system. In the following, we will refer to the different regions as "bridge" and "bulk." The width $W$ of one of the trenches is $230 \mathrm{~nm}$ and the length $L=1040 \mathrm{~nm}$. A thin residual $\mathrm{Ge}$ hemline along the bridge borders remained, whose origin is linked to the protective coating that was applied during the bridge suspension.

A common method for the investigation of the effects of strain in the $\mathrm{Si} / \mathrm{Ge}$ material system is micro-Raman spectroscopy, in which the shift of the scattered Raman signal is linked to the strain in the material. ${ }^{32-34}$ We used a microRaman set-up in backscattering geometry equipped with a $100 \times 0.9$ NA objective and $0.1 \mu \mathrm{m}$ step positioning stage. A frequency-doubled Nd:YAG laser with $532 \mathrm{~nm}$ output wavelength was used, producing a spot size less than $1 \mu \mathrm{m}$ on the sample. The choice of the excitation wavelength is justified by the vicinity of $532 \mathrm{~nm}$ wavelength to the Raman resonance in $\mathrm{Ge}^{35}$ so that we could use a power of $0.05 \mathrm{~mW}$, low enough to avoid heating artefacts in these structures characterized by low thermal dissipation. The excitation was linearly polarized along $y$, while the collection was not polarized. With this experimental geometry, only the longitudinal-optical (LO) phonon can be probed. Even though in principle also transverse-optical (TO) modes could be collected through the marginal rays of an objective with high numerical aperture, their intensity is expected to be negligible with this unpolarized collection geometry. ${ }^{36}$ The system was calibrated with a reference bulk crystal to the GeGe phonon mode at $\omega_{0}=300.3 \mathrm{~cm}^{-1}$. The Raman shift value $\omega$ of the strained material was obtained from a fit of the acquired Raman spectrum to a simple model described in detail in the next paragraph. Since for symmetry the offdiagonal strain components are negligible, strain values $\varepsilon$ were determined by setting up the strain tensor and using the relationship

$$
\Delta \omega=\omega-\omega_{0}=\frac{1}{2 \omega_{0}}\left(q \varepsilon_{x x}+q \varepsilon_{y y}+p \varepsilon_{z z}\right),
$$

where $q$ and $p$ are the deformation potentials for $\mathrm{Ge}$ as defined in Ref. 37. Information about the strain state of the Ge beneath a stressor can be obtained from the shift of its Raman band. Since the spot size of the scattered laser on the sample is larger than the width of the arms of the cross stressor and the $50 \mathrm{~nm}$ thick SiGe top layer is transparent at $532 \mathrm{~nm}$ wavelength (the penetration depth in SiGe with $60 \%$ Ge is estimated to be $\left.\delta_{\mathrm{SiGe}}=90 \mathrm{~nm}\right),{ }^{38}$ both SiGe layer and Ge layer are probed.

Let us first consider the unpatterned bulk, see Fig. 2(a). The Ge band in this region is centered at $300.1 \pm 0.1 \mathrm{~cm}^{-1}$. This value is slightly red-shifted with respect to the reference in accordance with a slight tensile thermal strain. ${ }^{39}$ The three prominent bands of the spectra originating from $\mathrm{SiGe}$ are related to the $\mathrm{Ge}-\mathrm{Ge}, \mathrm{Si}-\mathrm{Ge}$, and $\mathrm{Si}-\mathrm{Si} \mathrm{LO}$ modes. ${ }^{40}$ In this work, we will focus on the Ge-Ge mode since this is the most intense. The position of this band in the bulk is 
(a)

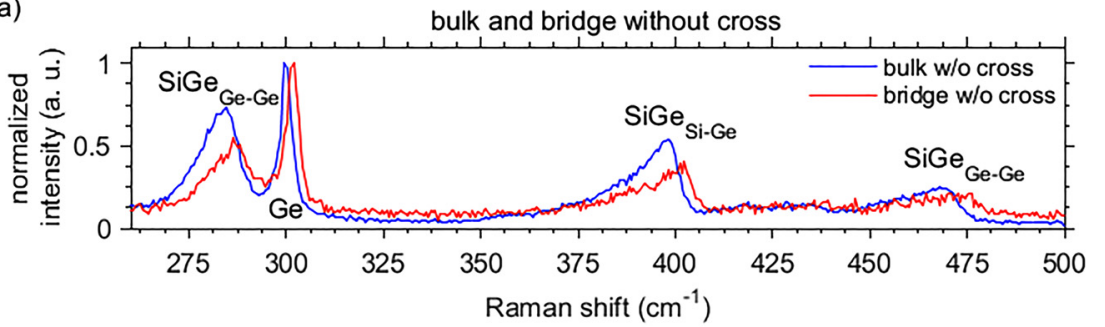

(b)

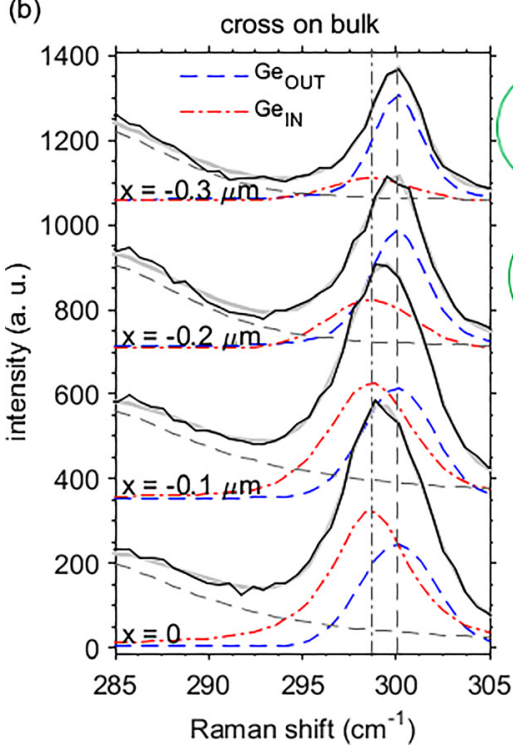

cross on bridge

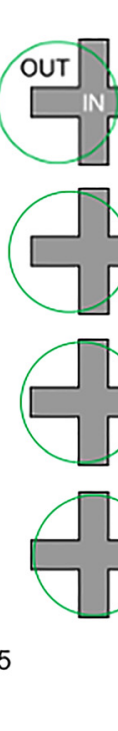

(c)

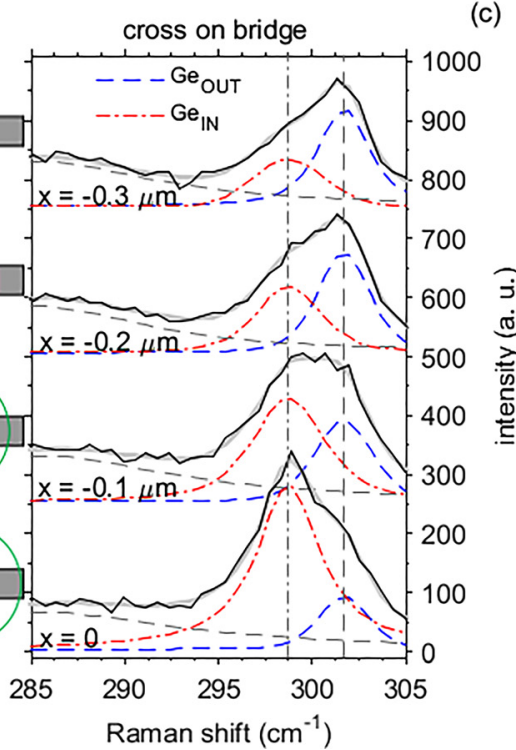

FIG. 2. (a) Raman spectra of the bulk without cross (blue curve) and an unpatterned reference bridge (red curve). The different visible modes are labeled. The Raman spectra obtained from a line scan across a cross stressor on the bulk and on the bridge along direction $x$ (cf. Fig. 1(c)) are shown in panels (b) and (c). The upper spectra of each series are taken from a position outside the center of the cross, whereas the position of the last spectra is in the center as indicated by the green circle in the sketches between panels (b) and (c). The data are fitted by the thick grey line, which is a combination of the single bands for Ge not altered by the cross stressor (blue dashed line), Ge altered by the stressor (red dasheddotted line), and the Ge-Ge mode of SiGe (grey dashed line).
$284.0 \pm 0.9 \mathrm{~cm}^{-1}$ which corresponds to $(1.6 \pm 0.2) \%$ tensile strain. Both of these values are in agreement with XRD. We can compare these results to those related to the unpatterned $\mathrm{SiGe} / \mathrm{Ge}$ bridge, cf. Fig. 2(a). Raman measurements on a suspended reference bridge without cross stressors revealed that the Ge layer is slightly compressed (Raman shift $\omega=301.7 \mathrm{~cm}^{-1}$, which corresponds to a strain of $-0.3 \%$ ) and that the $\mathrm{SiGe}$ layer is less tensile, i.e., partially relaxed, with Raman shift $\omega_{\mathrm{Ge}-\mathrm{Ge}}=287.1 \mathrm{~cm}^{-1}$ which corresponds to $1.1 \%$ strain. This change in the strain is readily explained by a redistribution of the strain in the bilayered bridge. Due to the removal of the mechanical constraint perpendicular to the bridge (direction $x$ in Fig. 1(c)) transverse elastic relaxation of tensile stress in the SiGe layer is significant and the reduction of the tensile strain field in the SiGe layer causes a compression of the Ge layer. However, it is important to notice that SiGe is still in a highly tensile state, which is the prerequisite for it to be used to create nanostructured stressors.

We now investigate the effect of etching crossed trenches into the bulk and the bridge. The results are presented in Figs. 2(b) and 2(c) for the bulk and bridge, respectively. With respect to the unpatterned case, these measurements are more critical since the nanostructures are of comparable size to the laser spot. For this reason, the effects related to the cross stressors can be better appreciated observing a line scan with steps of $0.1 \mu \mathrm{m}$ running from outside $(x=-0.3 \mu \mathrm{m})$ towards the center of the cross $(x=0)$, as depicted by the sketches in between the panels. Visual inspection of panel (b) shows a band at about $285 \mathrm{~cm}^{-1}$ from the SiGe stressor and a band at about $300 \mathrm{~cm}^{-1}$ from the Ge film. Moving towards the center of the cross, we can see that the position of the Ge band moves towards tensile values. Visual inspection of panel (c) suggests that the Ge band features two components, whose relative intensity changes as we approach the center of the cross. We interpret these two components as related to Ge outside the cross $\left(\mathrm{Ge}_{\mathrm{OUT}}\right)$ and $\mathrm{Ge}$ inside the cross $\left(\mathrm{Ge}_{\mathrm{IN}}\right)$. In the upper spectra, the main contribution to $\mathrm{Ge}$ is from $\mathrm{Ge}_{\mathrm{OUT}}$. As we approach the center of the cross, the $\mathrm{Ge}_{\mathrm{IN}}$ component from tensile Ge appears and overcomes the $\mathrm{Ge}_{\mathrm{OUT}}$ component. The separation of the band into two components can be observed only in the cross on the bridge because in this case the strain induced by the cross is much higher than the case of the cross on bulk.

In order to consistently extract quantitative data, we fitted all the spectra from both bulk and bridge with the same model consisting of three peaks representing $\mathrm{SiGe}, \mathrm{Ge}_{\text {OUT }}$, and $\mathrm{Ge}_{\mathrm{IN}}$. We use two components for Ge to model a complex distribution of strain because previous simulations ${ }^{29}$ suggest that, within the small penetration depth of the laser $(20 \mathrm{~nm})$, in these structures there are indeed two regions with well separated values of strain: Inside the cross and outside the cross. In addition, a higher number of components would lead to an uncontrolled fit. In both Figs. 2(b) and 2(c), the spectral position of the components was used as a global parameter for the simultaneous fitting of the spectra. For the bulk case, the fitted $\mathrm{Ge}_{\text {IN }}$ and $\mathrm{Ge}_{\text {OUT }}$ components are separated by $\Delta \omega=-1.4 \mathrm{~cm}^{-1}$, while for the bridge case they are separated by $\Delta \omega=-3.0 \mathrm{~cm}^{-1}$. As pointed out above, this increased splitting reflects the fact that on the bridge the strain induced by the cross is much higher than the case of the cross on bulk. For the bridge case, the SiGe can transfer more of its elastic energy to the underlying Ge so that the resulting strain is higher. ${ }^{29}$ 
We now proceed with the quantification of the strain. The cross stressors create biaxial strain in the center of the cross. However, the center of the cross, i.e., the intersection of the arms, is only $230 \times 230 \mathrm{~nm}^{2}$, too small to be resolved spatially. Since the area ratio of the center to the arms of the cross is about 1:6, what we detect with the Raman measurement stems mainly from the arms, which are under uniaxial strain. For the transformation of Raman shift into strain we have to consider the elements of the strain tensor given by Eq. (1)

$$
\begin{gathered}
\varepsilon_{x x}=\varepsilon_{\text {comp. bias }}+\varepsilon_{\text {therm }}+\varepsilon_{x x, \text { cross }}, \\
\varepsilon_{y y}=\varepsilon_{\text {therm }}+\varepsilon_{y y, \text { cross }}, \\
\varepsilon_{z z}=-\frac{c_{12}}{c_{11}}\left(\varepsilon_{x x}+\varepsilon_{y y}\right),
\end{gathered}
$$

$\varepsilon_{\text {comp.bias }}$ is the compressive strain bias along direction $x$, $\varepsilon_{\text {therm }}$ is the biaxial thermal strain originating in the growth of $\mathrm{Ge}$ on $\mathrm{Si}, \varepsilon_{x x, \text { cross }}$ and $\varepsilon_{y y, \text { cross }}$ are the strain induced by the cross stressor along directions $x$ and $y$, respectively, and $\mathrm{c}_{12}$ and $c_{11}$ are the elastic stiffness constants of Ge. The strain values are then obtained by solving Eq. (1).

Since $\varepsilon_{\text {comp.bias }}$ and $\varepsilon_{\text {therm }}$ are known from the independent measurements on the bulk and on the bridge, it is possible to calculate the strain in the material induced by the presence of the cross, $\varepsilon_{x x, \text { cross }}$, i.e., the additional strain relative to the Ge without crosses. Table I shows a summary of the results in terms of Raman shift and obtained uniaxial strain values for absolute and relative ("cross induced") strain, demonstrating that the stressor on the bridge is more efficient than that on the bulk material leading to twice the relative strain value. However, the strain induced by the stressors is nonuniform, because the stress forces act only at the perimeter of the cross, which means that the reported values give an average of the strain distribution. If we ascribe these average values to the arms and assume that each arm exerts an additive force on the center of the cross, it is possible to infer that the strain in the center of the cross will be biaxial and would reach at most $1.3 \%$; only $0.6 \%$ would be reached by the same nanostructure in the bulk case. This value is lower than the $1.6 \%$ biaxial strain required to obtain direct gap material. ${ }^{6}$ However, this method could represent an interesting alternative straining strategy, because optimization of stressor geometry and process parameters could lead to increased tensile strain, as predicted in Ref. 29.

In summary, we have fabricated cross-shaped $\mathrm{SiGe}$ stressors on Ge micro-bridges and bulk material. We showed that the Ge inside a stressor is under tensile strain, with the absolute strain values of the order of $0.7 \%$ uniaxial strain for both micro-bridge and bulk. For the symmetry of the system, a $0.7 \%$ biaxial strain is expected in the center of the cross.

TABLE I. Strain induced by the cross measured by Raman spectroscopy. $\omega_{\text {IN }}$ and $\omega_{\text {OUT }}$ are the spectral positions of the $\mathrm{Ge}_{\mathrm{IN}}$ and $\mathrm{Ge}_{\mathrm{OUT}}$ components. $\Delta \omega$ is the difference $\omega_{\mathrm{IN}}-\omega_{\mathrm{OUT}} \cdot \varepsilon_{\mathrm{xx}}$, cross is the strain induced by the cross, $\varepsilon_{\mathrm{Xx}}$ is the overall strain as defined in Eq. (2a).

\begin{tabular}{lccccc}
\hline \hline & $\begin{array}{c}\omega_{\text {OUT }} \\
\left(\mathrm{cm}^{-1}\right)\end{array}$ & $\begin{array}{c}\omega_{\mathrm{IN}} \\
\left(\mathrm{cm}^{-1}\right)\end{array}$ & $\begin{array}{c}\Delta \omega \\
\left(\mathrm{cm}^{-1}\right)\end{array}$ & $\begin{array}{c}\varepsilon_{\mathrm{xx}, \text { cross }} \\
(\%)\end{array}$ & $\begin{array}{c}\varepsilon_{\mathrm{Xx}} \\
(\%)\end{array}$ \\
\hline Bulk & $300.1 \pm 0.2$ & $298.7 \pm 0.7$ & $-1.4 \pm 0.7$ & $0.6 \pm 0.3$ & $0.7 \pm 0.3$ \\
Bridge & $301.7 \pm 0.2$ & $298.7 \pm 0.1$ & $-3.0 \pm 0.2$ & $1.3 \pm 0.1$ & $0.7 \pm 0.1$ \\
\hline \hline
\end{tabular}

Neglecting the compressive strain bias of the micro-bridge, which can be reduced by a proper development of the bridge, the obtained relative strain is $1.3 \%$ uniaxial on the arms and $1.3 \%$ biaxial in the center. This proves that creating nanostructures on top of the suspended material allows higher strains to be induced as compared to bulk material, due to sharing of the elastic energy. Previous calculations showed that in principle it is possible to raise these values by improving the fabrication, especially the etching step. The possibility of filling one bridge with several stressors could reduce the wafer footprint for several active areas. Our study broadens the discussion on how to create tensile strain in Ge to exploit this material as a light source for monolithic integration into Si technology.

We acknowledge Fondazione Cariplo (within the Grant DefCon4 2011-0331) for the financial support.

${ }^{1}$ G. T. Reed, G. Mashanovich, F. Y. Gardes, and D. J. Thomson, Nat. Photonics 4, 518 (2010).

${ }^{2}$ J. Michel, J. Liu, and L. C. Kimerling, Nat. Photonics 4, 527 (2010).

${ }^{3}$ P. Chaisakul, D. Marris-Morini, J. Frigerio, D. Chrastina, M.-S. Rouifed, S. Cecchi, P. Crozat, G. Isella, and L. Vivien, Nat. Photonics 8, 482 (2014).

${ }^{4}$ M. L. Lee, E. A. Fitzgerald, M. T. Bulsara, M. T. Currie, and A. Lochtefeld, J. Appl. Phys. 97, 011101 (2005).

${ }^{5}$ T. P. McLean, Progress in Semiconductors, edited by A. F. Gibson (Heywood, London, 1960), Vol. 5, p.53.

${ }^{6}$ M. Virgilio, C. L. Manganelli, G. Grosso, G. Pizzi, and G. Capellini, Phys. Rev. B 87, 235313 (2013).

${ }^{7}$ M. V. Fischetti and S. E. Laux, J. Appl. Phys. 80, 2234 (1996).

${ }^{8}$ J. Liu, X. Sun, D. Pan, X. Wang, L. C. Kimerling, T. L. Koch, and J. Michel, Opt. Express 15, 11272 (2007).

${ }^{9}$ J. Liu, X. Sun, L. C. Kimerling, and J. Michel, Opt. Lett. 34, 1738 (2009).

${ }^{10}$ J. Liu, X. Sun, R. Camacho-Aguilera, L. C. Kimerling, and J. Michel, Opt. Lett. 35, 679 (2010).

${ }^{11}$ R. E. Camacho-Aguilera, Y. Cai, N. Patel, J. T. Bessette, M. Romagnoli, L. C. Kimerling, and J. Michel, Opt. Express 20, 11316 (2012).

${ }^{12}$ R. Koerner, M. Oehme, M. Gollhofer, M. Schmid, K. Kostecki, S. Bechler, D. Widmann, E. Kasper, and J. Schulze, Opt. Express 23, 14815 (2015).

${ }^{13}$ Y. Ishikawa, K. Wada, D. D. Cannon, J. Liu, H.-C. Luan, and L. C. Kimerling, Appl. Phys. Lett. 82, 2044 (2003).

${ }^{14}$ L. Carroll, P. Friedli, S. Neuenschwander, H. Sigg, S. Cecchi, F. Isa, D. Chrastina, G. Isella, Y. Fedoryshyn, and J. Faist, Phys. Rev. Lett. 109, 057402 (2012).

${ }^{15}$ B. Dutt, D. Sukhdeo, D. Nam, B. Vulovic, Z. Yuan, and K. Saraswat, Photonics J. 4, 2002 (2012).

${ }^{16}$ D. Sukhdeo, D. Nam, Z. Yuan, B. (R.) Dutt, and K. C. Saraswat, "Toward an efficient germanium-on-silicon laser: Ultimate limits of tensile strain and n-type doping," in CLEO: 2013, OSA Technical Digest (Optical Society of America, 2013), Paper No. JTh2A.109.

${ }^{17}$ M. Virgilio, C. L. Manganelli, G. Grosso, T. Schroeder, and G. Capellini, J. Appl. Phys. 114, 243102 (2013).

${ }^{18}$ A. Ghrib, M. de Kersauson, M. El Kurdi, R. Jakomin, G. Beaudoin, S. Sauvage, G. Fishman, G. Ndong, M. Chaigneau, R. Ossikovski, I. Sagnes, and P. Boucaud, Appl. Phys. Lett. 100, 201104 (2012).

${ }^{19}$ G. Capellini, G. Kozlowski, Y. Yamamoto, M. Lisker, C. Wenger, G. Niu, P. Zaumseil, B. Tillack, A. Ghrib, M. de Kersauson, M. El Kurdi, P. Boucaud, and T. Schroeder, J. Appl. Phys. 113, 013513 (2013).

${ }^{20}$ M. Bollani, D. Chrastina, L. Gagliano, L. Rossetto, D. Scopece, M. Barget, V. Mondiali, J. Frigerio, M. Lodari, F. Pezzoli, F. Montalenti, and E. Bonera, Appl. Phys. Lett. 107, 083101 (2015).

${ }^{21}$ D. Nam, D. Sukhdeo, A. Roy, K. Balram, S.-L. Cheng, K. C.-Y. Huang, Z. Yuan, M. Brongersma, Y. Nishi, D. Miller, and K. Saraswat, Opt. Express 19, 25866 (2011).

${ }^{22}$ A. Ghrib, M. El Kurdi, M. de Kersauson, M. Prost, S. Sauvage, X. Checoury, G. Beaudoin, I. Sagnes, and P. Boucaud, Appl. Phys. Lett. 102, 221112 (2013).

${ }^{23}$ G. Capellini, C. Reich, S. Guha, Y. Yamamoto, M. Lisker, M. Virgilio, A. Ghrib, M. E. Kurdi, P. Boucaud, B. Tillack, and T. Schroeder, Opt. Express 22, 399 (2014). 
${ }^{24}$ V. Mondiali, M. Lodari, D. Chrastina, M. Barget, E. Bonera, and M. Bollani, Microelectron. Eng. 141, 256 (2015).

${ }^{25}$ M. J. Süess, R. Geiger, R. A. Minamisawa, G. Schiefler, J. Frigerio, D. Chrastina, G. Isella, R. Spolenak, J. Faist, and H. Sigg, Nat. Photonics 7, 466 (2013).

${ }^{26}$ D. S. Sukhdeo, D. Nam, J.-H. Kang, M. L. Brongersma, and K. C. Saraswat, Photonics Res. 2, A8 (2014).

${ }^{27}$ G. Dias, D. Rouchon, J. Widiez, J. Hartmann, D. Fowler, A. Chelnokov, V. Reboud, A. Gassenq, S. Tardif, K. Guiloy, N. Pauc, J. Escalante, I. Duchemin, Y.-M. Niquet, F. Rieutord, V. Calvo, J. Faist, R. Geiger, T. Zabel, and H. Sigg, in 2015 IEEE 12th International Conference on Group IV Photonics (GFP), Vancouver, BC (2015), pp. 133-134.

${ }^{28}$ A. Gassenq, K. Guilloy, G. Osvaldo Dias, N. Pauc, D. Rouchon, J.-M. Hartmann, J. Widiez, S. Tardif, F. Rieutord, J. Escalante, I. Duchemin, Y.-M. Niquet, R. Geiger, T. Zabel, H. Sigg, J. Faist, A. Chelnokov, V. Reboud, and V. Calvo, Appl. Phys. Lett. 107, 191904 (2015).

${ }^{29}$ D. Scopece, F. Montalenti, E. Bonera, M. Bollani, and D. Chrastina, Semicond. Sci. Technol. 29, 095012 (2014).

${ }^{30}$ V. Mondiali, M. Lodari, M. Borriello, D. Chrastina, and M. Bollani, Microelectron. Eng. 153, 88 (2016).
${ }^{31}$ J. Frigerio, M. Lodari, D. Chrastina, V. Mondiali, G. Isella, and M. Bollani, J. Appl. Phys. 116, 113507 (2014).

${ }^{32}$ F. Pezzoli, E. Bonera, E. Grilli, M. Guzzi, S. Sanguinetti, D. Chrastina, G. Isella, H. von Känel, E. Wintersberger, J. Stangl, and G. Bauer, J. Appl. Phys. 103, 093521 (2008).

${ }^{33}$ J. S. Reparaz, A. Bernardi, A. R. Goñi, M. I. Alonso, and M. Garriga, Appl. Phys. Lett. 92, 081909 (2008).

${ }^{34}$ E. Bonera, M. Bollani, D. Chrastina, F. Pezzoli, A. Picco, O. G. Schmidt, and D. Terziotti, J. Appl. Phys. 113, 164308 (2013).

${ }^{35}$ A. Picco, E. Bonera, E. Grilli, M. Guzzi, M. Giarola, G. Mariotto, D. Chrastina, and G. Isella, Phys. Rev. B 82, 115317 (2010).

${ }^{36}$ E. Bonera, M. Fanciulli, and D. N. Batchelder, Appl. Phys. Lett. 81, 3377 (2002).

${ }^{37}$ F. Cerdeira, C. Buchenauer, F. Pollak, and M. Cardona, Phys. Rev. B 5, 580 (1972).

${ }^{38}$ J. Humlíček, M. Garriga, M. I. Alonso, and M. Cardona, J. Appl. Phys. 65, 2827 (1989).

${ }^{39}$ F. Pezzoli, E. Bonera, E. Grilli, M. Guzzi, S. Sanguinetti, D. Chrastina, G. Isella, H. von Känel, E. Wintersberger, J. Stangl, and G. Bauer, Mater. Sci. Semicond. Process. 11, 279 (2008).

${ }^{40}$ M. I. Alonso and K. Winer, Phys. Rev. B 39, 10056 (1989). 